\title{
Guest-editorial
}

\section{Special issue recent advances in soft computing: Theories and applications}

\author{
Chee Peng Lim ${ }^{\mathrm{a}, *}$, Valentina Emilia Balas ${ }^{\mathrm{b}}$ and Quoc Do ${ }^{\mathrm{c}}$ \\ ${ }^{a}$ Deakin University, Burwood, VIC, Australia \\ b "Aurel Vlaicu” University of Arad, Arad, Romania \\ ${ }^{\mathrm{c}}$ University of South Australia, Mawson Lakes, SA, Australia
}

\begin{abstract}
Soft Computing is an interdisciplinary area that encompasses a variety of computing paradigms. Examples of some popular soft computing paradigms include fuzzy computing, neural computing, evolutionary computing, and probabilistic computing. Soft computing paradigms, in general, aim to produce computing systems/machines that exhibit some useful properties, e.g. making inference with vague and/or ambiguous information, learning from noisy and/or incomplete data, adapting to changing environments, and reasoning with uncertainties. These properties are important for the systems/machines to be useful in assisting humans in our daily activities. Indeed, soft computing paradigms have been demonstrated to be capable of tackling a wide range of problems, e.g. optimization, decision making, information processing, pattern recognition, and intelligent data analysis. A number of papers pertaining to some recent advances in theoretical development and practical application of different soft computing paradigms are highlighted in this special issue.
\end{abstract}

\section{Introduction}

The aim of this special issue is to showcase a small fraction of recent advances in terms of theory and application of soft computing paradigms. A total of six papers are included in this special issue. It is by no mean exhaustive as this is a fast-moving area in which new principles and applications of soft computing paradigms emerge almost daily. A summary of each paper is as follows.

An attempt to determine the optimal number of clusters for a data set is described in the first paper [5]. Two independent sample sets, one is drawn from the original data set and another from a noisy version of the data set, are formed. The partitions built from these two sample sets are compared using the Binomial $k$-nearest neighbor model. The quality of a cluster partition is

\footnotetext{
${ }^{*}$ Corresponding author. Chee Peng Lim, Deakin University, Burwood, VIC, Australia. E-mail: chee.lim@deakin.edu.au.
}

characterized using indexes based on the $p$-values. A series of experimental studies demonstrate the effectiveness of the proposed method as compared with those from several other cluster validation methods.

A method that uses observations from the analysis of attributes for optimizing rule-based and neural networkbased classifiers is described in the second paper [3]. The frequencies of the attributes in decision rules with varying lengths are analyzed, and useful relevance measures are computed and are used for attribute/feature reduction. Experimental results show the effectiveness of the proposed method in estimating relevant attributes for constructing rule-based and neural network-based classifiers.

In the third paper [6], the properties of linguistic truth-valued intuitionistic fuzzy lattice are discussed. In particular, the relationship between linguistic truthvalued intuitionistic fuzzy lattice and other logical algebras are analyzed. It is shown that the linguistic 
truth-valued intuitionistic fuzzy lattice is able to deal with positive and negative evidences, as well as to better express and handle both comparable and incomparable information.

A rule-based method to assess the situation awareness of a pilot, i.e., the ability to perceive information from an environment and to detect if any problem exists in the environment, is presented in the fourth paper [1]. Eye movements of a pilot are monitored continuously. Based on the attentional distribution patterns of eye movements, the behaviors of expert and novice pilots in controlling an aircraft are analyzed and differentiated. From the analysis, potential loss of situation awareness by novice pilots could be detected, and a warning signal could be issued to avoid human errors in flight operations.

In the fifth paper [2], modeling of a six-legged robot and prediction of two outputs, i.e., the specific energy consumption and normalized energy stability margin, with respect to crab walking of the robot are described. A number of adaptive neuro-fuzzy inference systems tuned by back-propagation and genetic algorithms are deployed, with the performances compared and analyzed. The results indicate that the genetic algorithm-tuned multiple adaptive neuro-fuzzy inference system is able to produce the best predictions in terms of the two output indicators.

A modified micro genetic algorithm to tackle multiobjective optimization problems is proposed in the last paper [4]. Strategies for elitism selection and population initialization are incorporated into the conventional micro genetic algorithm, with the aim to accelerate convergence of the solutions towards the pareto optimal front. Experimental results using benchmark problems demonstrate the usefulness of the proposed method in producing solutions with improved generational distance for undertaking multi-objective optimization problems.

The guest editors would like to express sincere gratitude and appreciation to (i) the authors for their contributions to this special issue; (ii) the reviewers for their hard work in assessing the articles within a short timeframe; and (iii) the members of the production team for producing this special issue.

\section{References}

[1] K. Kilingaru, J.W. Tweedale, S. Thatcher and L.C. Jain, Monitoring pilot situation awareness, Journal of Intelligent \& Fuzzy Systems 24 (2013), 457-466.

[2] S.S. Roy and D.K. Pratihar, Adaptive neuro-fuzzy expert systems for predicting specific energy consumption and energy stability margin in crab walking of six-legged robots, Journal of Intelligent \& Fuzzy Systems 24 (2013), 467-482.

[3] U. Stanczyk, Decision rule length as a basis for evaluation of attribute relevance, Journal of Intelligent \& Fuzzy Systems $\mathbf{2 4}$ (2013), 429-445.

[4] C.J. Tan, C.P. Lim and Y-N Cheah, A modified micro genetic algorithm for undertaking multi-objective optimization problems, Journal of Intelligent \& Fuzzy Systems 24 (2013), 483-495.

[5] D. Toledano-Kitai, R. Avros, Z. Volkovich, G-W. Weber and O. Yahalom, A binomial noised model for cluster validation, Journal of Intelligent \& Fuzzy Systems 24 (2013), 417-427.

[6] L. Zou, P. Shi, Z. Peix and Y. Xu, On an algebra of linguistic truth-valued intuitionistic lattice-valued logic, Journal of Intelligent \& Fuzzy Systems 24 (2013), 447-456. 